\title{
Signature of Two-Electron Interference in Angular Resolved Double Photoionization of Mg
}

\author{
E. Sokell, ${ }^{1}$ P. Bolognesi, ${ }^{2}$ A. Kheifets, ${ }^{3}$ I. Bray,${ }^{4}$ S. Safgren, ${ }^{1}$ and L. Avaldi ${ }^{2}$ \\ ${ }^{1}$ School of Physics, UCD Science Centre, Belfield, Dublin 4, Ireland \\ ${ }^{2}$ CNR-Istituto di Metodologie Inorganiche e dei Plasmi, Area della Ricerca di Roma 1, 00015 Monterotondo Scalo, Italy \\ ${ }^{3}$ RSPE, The Australian National University, Canberra ACT 0200, Australia \\ ${ }^{4}$ ARC Centre for Antimatter-Matter Studies, Curtin University, Perth, Western Australia 6845, Australia \\ (Received 16 July 2012; revised manuscript received 25 October 2012; published 20 February 2013)
}

\begin{abstract}
The double photoionization of $\mathrm{Mg}$ has been studied experimentally and theoretically in a kinematic where the two photoelectrons equally share the excess energy. The observation of a symmetrized gerade amplitude, which strongly deviates from the Gaussian ansatz, is explained by a two-electron interference predicted theoretically, but never before observed experimentally. Similar to the Cooper minima in the single photoionization cross section, the effect finds its origin in the radial extent and oscillation of the target wave function.
\end{abstract}

DOI: 10.1103/PhysRevLett.110.083001

PACS numbers: $32.80 . \mathrm{Fb}$

The effect of the target orbital on the photoionization cross section in the form of the Cooper minimum has long been established [1] and commonly used to obtain information on the electronic structure in the gas phase [2] as well as in condensed matter [3,4]. The Cooper minimum, observed for all the orbitals with a number of nodes $n-\ell-1>0$, is determined by the vanishing radial overlap between the photoelectron wave function and the target orbital. Generally there are the two photoionization channels with photoelectron angular momenta $\ell \pm 1$, the node in the stronger channel is offset by a nonvanishing contribution in the weaker channel. The situation is much more complicated in double photoionization (DPI) where there are no simple bounds on the individual photoelectron angular momenta $\ell_{1}$ and $\ell_{2}$ other than coupling to the total angular momentum of the pair, and a large number of radial integrals contribute to the total integrated photoionization cross section. Hence, there is no simple analogue of the Cooper minimum in DPI.

Nevertheless, a strong effect of the target electronic structure was observed in calculations of the angular correlation pattern in the two-electron continuum [5]. It has been shown that (i) for a given symmetry of the electron pair the angular correlation of DPI mimics the angular distribution of an $e$-impact ionization of the corresponding ion and (ii) the amplitudes of these processes are strongly determined by the radial extent and oscillations of the target orbital of the singly charged ion. In the case of the Cooper minimum, the contributions of the positive and negative oscillations of the target orbital cancel each other in the real and scalar quantity (the dipole radial integral). In the case of DPI, these contributions add up as complex and angular dependent amplitudes. They do not vanish entirely, but display an intricate interference pattern.

For a long time, investigation of DPI was focused on the $\mathrm{He}$ atom, the simplest two-electron system [6-8]. Because of a nodeless $1 s$ target orbital, the dynamic DPI amplitude of this process (also known as correlation factor [9]) showed a very simple Gaussian shape. Alkaline-earth-metal atoms (Be, $\mathrm{Mg}, \mathrm{Ca}, \mathrm{Sr})$ are "quasi-two-electron" systems with the outermost orbitals characterized by one or more nodes and represent the most suitable candidates for extending the investigation of DPI beyond He. The theoretical DPI cross section of Be and heavier alkaline-earth-metal atoms has been calculated within several computational schemes [10-16]. A systematic investigation by Kheifets and Bray [17] elucidated the role of the ground- and finalstate correlations and showed that the narrowing of the angular correlation pattern in the triple differential cross section (TDCS), as the mass of the alkali-earth element increases, is related to a more diffuse $n s$ target orbital bound to the singly charged ion.

Stimulated by these novel theoretical predictions we have undertaken an investigation of the DPI of $\mathrm{Mg}$ to $\mathrm{Mg}^{2+}\left(3 s^{-2}\right)$ in the equal energy sharing conditions at $55.49 \mathrm{eV}$. The experiments have been performed using the multicoincidence end station [18] of the Gas Phase Photoemission beam line [19] at Elettra, where an undulator of period $12.5 \mathrm{~cm}, 4.5 \mathrm{~m}$ long produces fully linearly polarized radiation in the photon energy range $13-1000 \mathrm{eV}$. The vacuum chamber hosts two independent turntables, holding respectively three and seven electrostatic hemispherical analyzers at $30^{\circ}$ with respect each other. The three spectrometers of the smaller turntable, are mounted at $0^{\circ}, 30^{\circ}$, and $60^{\circ}$ with respect to the polarization vector $\boldsymbol{e} \| \boldsymbol{x}$ of the light in the plane $(\mathbf{x}, \mathbf{y})$ perpendicular to the direction $\mathbf{z}$ of propagation of the radiation. They have been used to measure the "fixed electron," labeled 1. The larger turntable rotates in the same plane and its seven analyzers have been used to measure the angular distribution of the correlated electron, labeled 2 [20]. The ten analyzers have been set to detect electrons of kinetic energy $E_{1}=$ $E_{2}=16.4 \mathrm{eV}$. The energy resolution and the angular acceptance were $\Delta E / E_{1,2}=0.03$ and $\Delta \theta_{1,2}= \pm 3^{\circ}$, respectively. 
The photon energy resolution was about $150 \mathrm{meV}$. The relative angular efficiency of the ten analyzers has been established by measuring the photoelectron angular distributions of $\mathrm{Mg} 2 p$ and $\mathrm{Ne} 2 p$, with well-known asymmetry parameters. As in Ref. [21], the metal vapor source is collinear with the photon beam, which passes through the hollow core of the source before interacting with the atomic beam and ending up on the photodiode, to be monitored. Six apertures drilled into the closure piece of the crucible and pointing to the interaction region increase the atom density at the interaction region. The oven has been operated at a temperature setting of 410 and $470{ }^{\circ} \mathrm{C}$ for the bottom and top parts of the crucible, respectively. An independent hypodermic needle is used to admit rare gases in the interaction region for tuning and calibration purposes. An accumulation time of about $3 \mathrm{~h} /$ point was necessary to reach the present accuracy in the experimental results. The measurement of the TDCS of metal vapors even at the third generation synchrotron radiation sources is very challenging. Apart from one case in $\mathrm{Ca}$ [22], all the other measurements of the TDCS in alkaline-earth-metal atoms [23-26] have been made at energies corresponding to $n p \rightarrow n^{\prime} d$ resonances in the double continuum. Here the TDCS have been measured at $55.49 \mathrm{eV}$, which corresponds to the excitation of the $\operatorname{Mg}\left(2 p^{6} 3 s^{2}\right) \rightarrow \operatorname{Mg}\left(2 p^{5} 3 s^{2} 3 d\right)$ resonance. While, on one hand, the resonance enhances the photoabsorption cross section, on the other hand, it may affect the shape of the TDCS. Indeed the observation of extra features in previous measurements of the TDCS of alkaline-earth-metal atoms [23-26] with respect to $\mathrm{He}$ was interpreted as a signature of an indirect process, where the DPI proceeds via an intermediate neutral excited state.

The two-electron convergent close coupling (CCC) formalism [17] cannot tackle resonant processes $a b$ initio. Thus, to calculate the TDCS properly, the effect of the resonant excitation has been incorporated semi-empirically in the CCC formalism [20]. In doing so, we note that the dipole matrix element varies near the resonance as [27]

$$
D(\omega)=\frac{q+\varepsilon}{i+\varepsilon} D_{0} \simeq-i q D_{0}
$$

at $\omega \simeq \omega_{0}$ and $\varepsilon \simeq 0$. Here $\varepsilon=\left(\omega-\omega_{0}\right) /(\Gamma / 2)$ is the energy counted from the resonance and measured in units of its width, $q$ is the Fano profile index and $D_{0}$ is the dipole matrix element in the absence of the resonance. Equation (1) leads to the Fano formula for the cross section [28]

$$
\sigma(\omega)=\frac{(q+\varepsilon)^{2}}{1+\varepsilon^{2}} \sigma_{0} \simeq q^{2} \sigma_{0} .
$$

In principle, the resonance is present in all energetically accessible final channels of photoionization as well as in the double ionization channel. Each channel is characterized by its own profile index. For the $2 p \rightarrow 3 d$ resonance in $\mathrm{Mg}$, the profile indexes known from the singly and doubly charged total ion yields [29] and calculated for the lowest ionic state [30] are in the range between -40 and -50 . In the present calculation, we consider only the resonance in the ground ionic state, which makes the strongest contribution.

The experimental results, as well as the CCC calculations are shown on the left set of panels of Fig. 1 for four fixed reference angles $\theta_{1}$. For the sake of comparison, on the right set of panels of the figure, we show a similar set of TDCS of He at the equal energy sharing of $E_{1}=E_{2}=$ $10 \mathrm{eV}$ [31]. The evolution of the TDCS shape with $\theta_{1}$ displays similarities and differences between $\mathrm{He}$ and $\mathrm{Mg}$. In both cases, a node at $\theta_{12}=180^{\circ}$, as expected by the singlet odd character of the double continuum wave function, is observed at all $\theta_{1}$. However the lobes in $\mathrm{Mg}$ are significantly narrower and the relative intensity and number of minor lobes at $\theta_{1}=30^{\circ}$ and $60^{\circ}$ are different. A general good agreement is observed, within the

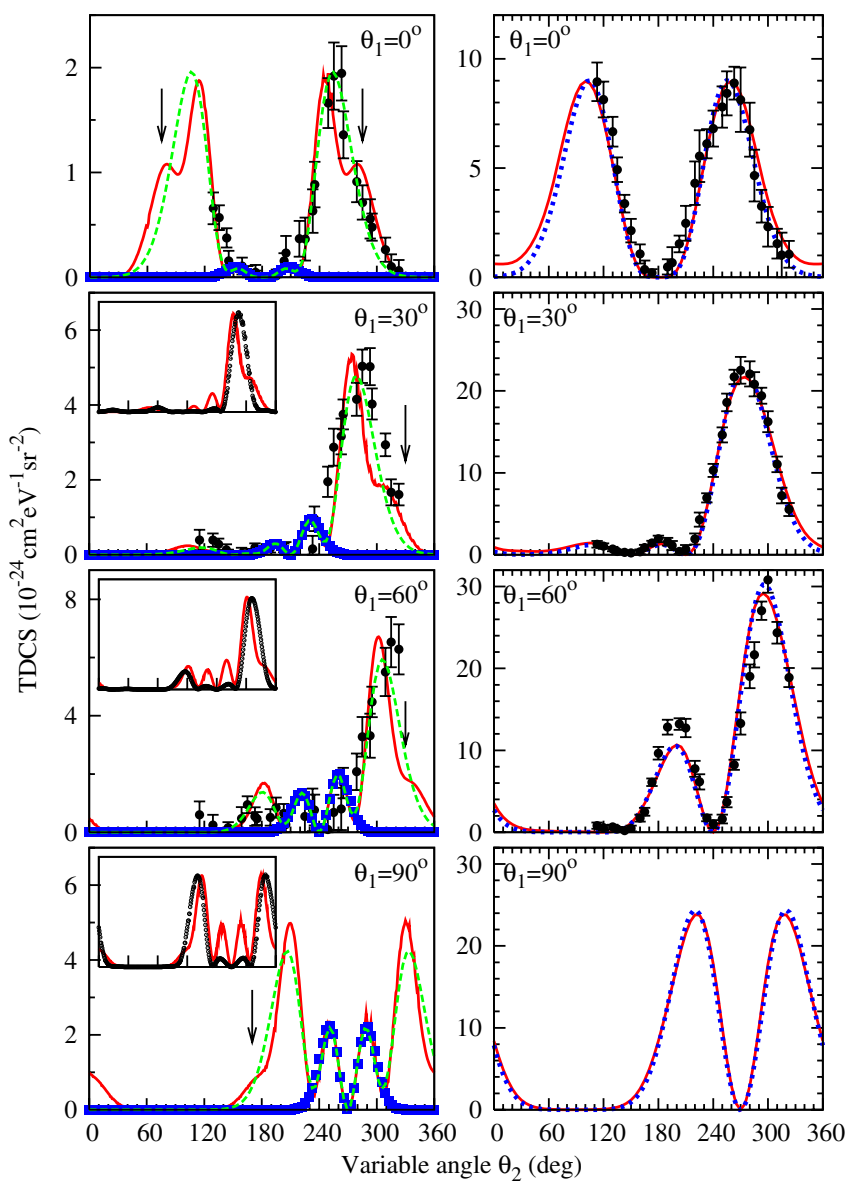

FIG. 1 (color online). Left: TDCS of DPI of Mg at $E_{1}=E_{2}=$ $16.4 \mathrm{eV}$ and various fixed reference $a \theta_{1}$. The resonant CCC calculation multiplied by the factor $q^{-2}$ (red solid line) is fitted with the Gaussian ansatz Eq. (4) (blue squares) and di-Gaussian parametrization Eq. (5) (green dashed line). The nonresonant CCC calculation (scaled to the resonant CCC calculation) is shown in the inset with the black dots. The experimental TDCS (shown with error bars) have been rescaled to $\mathrm{CCC}$ (see text). Right: TDCS of $\mathrm{He}$ at $E_{1}=E_{2}=10 \mathrm{eV}$ [31]; the CCC calculation (red solid line) is fitted with the Gaussian ansatz eq. (4) (blue dotted line). 
experimental uncertainties, between the experiment and the CCC predictions. The experimental TDCS at $\theta_{1}=$ $30^{\circ}$ and $60^{\circ}$ are mainly concentrated in one lobe, while theory predicts a small lobe at about $230^{\circ}$, a nonvanishing cross section at about $100^{\circ}$ at $\theta_{1}=30^{\circ}$ and a series of three small lobes at $\theta_{1}=60^{\circ}$. The quality of the data does not allow us to properly resolve all these features, but the general trend is consistent with the predictions. At all $\theta_{1}$ the additional feature (see arrows on the left set of panels of Fig. 1) predicted in the main lobe, cannot be discerned in the experimental data. As the three TDCS were measured simultaneously, they can be reported on the same relative scale. A common scaling factor between theory and experiment has been used for the TDCS of Mg at $\theta_{1}=30^{\circ}$ and $60^{\circ}$, while the theory appears to overestimate the experiment by a factor 2.2 at $\theta_{1}=0^{\circ}$. Similar variation of the scaling coefficients by a factor of 1.6 was required for He which indicates a level of agreement between the present theory and experiment that we may expect. The nonresonant $\mathrm{CCC}$ calculations are shown in the inset of the figures, where they are compared to the ones including the resonance. For this purpose, the two calculations are scaled in a way that the maxima match in each figure. The nonresonant CCC displays the same narrowing of the lobes and presence of extra features as in the TDCS, but for the ones indicated by the arrows in Fig. 1. The absolute cross section is about a factor of 4 lower than in the calculations including the resonance (divided by the $q^{2}$ factor) and the main lobes have an intensity enhanced with respect to the other features.

Under the equal energy sharing conditions, the TDCS is determined by the symmetric or gerade amplitude [6]

$$
\frac{d^{3} \sigma}{d \Omega_{1} d \Omega_{2} d E_{2}}=\left|\left[\boldsymbol{e} \cdot \boldsymbol{n}_{1}+\boldsymbol{e} \cdot \boldsymbol{n}_{2}\right] \mathcal{M}^{g}\left(\theta_{12}\right)\right|^{2},
$$

where the unit vectors $\boldsymbol{n}_{1}, \boldsymbol{n}_{2}$, and $\boldsymbol{e}$ denote escape directions of the photoelectrons and the polarization vector of light, respectively. The symmetric $M^{g}$ amplitudes for $\mathrm{Mg}$ and $\mathrm{He}$ are shown on the top and middle panels of Fig. 2, respectively.

The central portion of the $\mathrm{Mg}$ amplitude near the mutual photoelectron angle $\theta_{12}=180^{\circ}$ can be represented by the Gaussian ansatz

$$
\left|\mathcal{M}^{g}\left(\theta_{12}\right)\right| \approx A e^{-2 \ln 2\left(\pi-\theta_{12} / \Delta \theta\right)^{2}} \equiv G\left(A, \Delta \theta, \theta_{12}\right) .
$$

The same ansatz can be applied to the whole of the He amplitude. The He amplitude is well described by a Gaussian function centered at $\theta_{12}=180^{\circ}$, while only the portion of the $\mathrm{Mg}$ amplitude near $180^{\circ}$ can be represented by the Gaussian function. However, the fringes of the $\mathrm{Mg}$ amplitude at larger mutual angles $\theta_{12}$ violate the Gaussian ansatz. The whole of the $\mathrm{Mg}$ amplitude can be better described by the di-Gaussian parametrization proposed in Ref. [5]
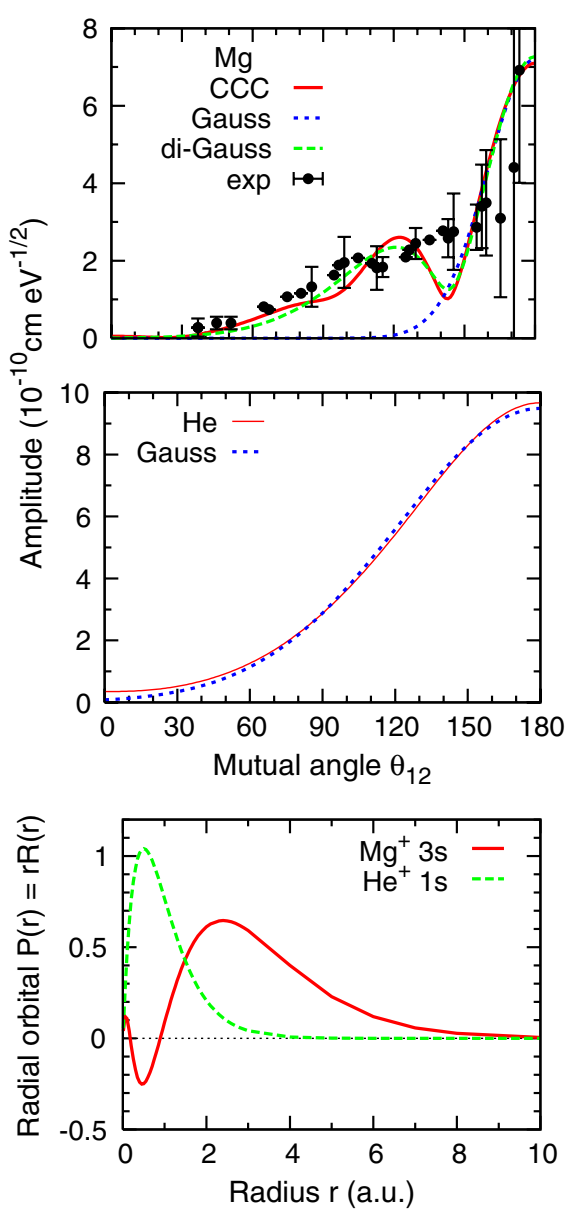

FIG. 2 (color online). The symmetric gerade amplitudes of DPI of $\mathrm{Mg}$ at $E_{1}=E_{2}=16.4 \mathrm{eV}$ (top) and $\mathrm{He}$ at $E_{1}=E_{2}=$ $10 \mathrm{eV}$ (middle) are drawn as functions of the mutual photoelectron angle $\theta_{12}$. The full CCC calculation (red solid line) is fitted with the Gaussian ansatz Eq. (4) (blue dashed line) and di-Gaussian parametrization Eq. (5) (green dashed line). The $\mathrm{Mg}$ amplitude extracted from the experimental TDCS is shown with error bars. Bottom: The radial orbitals $P(r)=r R(r)$ for $\mathrm{Mg}^{+} 3 s$ (red solid line) and $\mathrm{He}^{+} 1 s$ (green dashed line).

$\mathcal{M}^{g}\left(\theta_{12}\right)=G\left(A_{1}, \Delta \theta_{1}, \theta_{12}\right)+e^{i \phi} G\left(A_{2}, \Delta \theta_{2}, \theta_{12}\right)$

The complex phase factor represents the interference of the two Gaussians. The five constants $A_{1,2}, \Delta \theta_{1,2}$, and $\phi$ are used as fitting parameters. As was argued in Ref. [5], the Gaussian width may be linked to the radial extent of the target orbital bound to the singly charged ion. A more sparse target orbital can be reached by a larger number of partial waves of the electron in the continuum, which leads to a narrower Gaussian. Thus, it is natural to associate the wide and narrow Gaussians with two characteristic regions in the target coordinate space. In DPI of the $2 s$-shell atomic targets, these two regions are related to the positive and negative oscillations of the target orbital. In the present case of a $3 s$-shell target orbital, there are three oscillations but the first one, near the origin, is very small as is seen on 
the bottom panel of Fig. 2. So the di-Gaussian parameterization can be used. At the same time, the radial orbital bound to the $\mathrm{He}^{+}$ion has only one area of charge localization and thus, according to the arguments of Ref. [5], the corresponding DPI amplitude can be represented by just a single Gaussian.

The $\mathrm{Mg}$ amplitude, extracted from the experimental TDCS by Eq. (3), is also shown in the top panel of Fig. 2. The parameters of the di-Gaussian fit, Eq. (5), to the experimental data (and to the CCC calculations) are as follows: $A_{2} / A_{1}=0.42 \pm 0.08$ (0.43), $\Delta \theta_{1}=30 \pm 2$ (41.2), $\Delta \theta_{2}=95 \pm 4$ (89.2), $\phi=133 \pm 5$ (160). In comparison, the theoretical Gaussian width for $\mathrm{He}$ is $97^{\circ}$ which is similar to the $\Delta \theta_{2}$ parameter of $\mathrm{Mg}$. This is consistent with the similar peak positions of the negative oscillation of the $\mathrm{Mg}^{+} 3 s$ orbital and the positive oscillation of the $\mathrm{He}^{+} 1 s$ orbital, both shown on the bottom panel of Fig. 2 . The comparison between the theoretical and experimental di-Gaussian parameters is quite satisfactory. The experimental width $\Delta \theta_{1}$ tends to be smaller than the predicted one. This may be due to the quality of the data, which also hampers a clear observation of the predicted minimum in the amplitude. Even though the di-Gaussian fringe is relatively insignificant in comparison with the central Gaussian peak, its contribution to the TDCS is actually dominant. This is shown in Fig. 1 where the TDCS at $\theta_{1}=0^{\circ}, 30^{\circ}$, and $60^{\circ}$ are plotted. At $\theta_{1}=0^{\circ}$, almost all of the intensity of the TDCS comes from the di-Gaussian fringe and almost none from the central Gaussian peak. The Gaussian contribution becomes more significant at the other fixed angles $\theta_{1}=30^{\circ}$ and $60^{\circ}$, but the di-Gaussian fringe still dominates the TDCS. This behavior can be easily understood because the Gaussian peak at $\theta_{12}=$ $180^{\circ}$, which corresponds to the back-to-back emission, is suppressed by the kinematic factor due to the dipole selection rules, while the di-Gaussian fringe is away from the kinematic node and its contribution is not damped.

Once the analysis is extended to $\theta_{1}=90^{\circ}$ (bottom panels of Fig. 1) one sees that both the resonant and nonresonant CCC calculations clearly produce four lobes in the TDCS and they are well represented by a di-Gaussian amplitude. Thus these results prove that the extra lobes with respect to the He $1 s$ observed in previous measurements on alkaline-earth-metal atoms [23-26] are produced by the structure of the initial state $n s$ wave function. The resonant intermediate state only enhances their intensity. This is the first experimental observation of a strong modification of the symmetric amplitude in the DPI of a $n s$ orbital with respect to the one of He. This modification is related to the structure of the radial wave function of the target orbital. It manifests itself in a non-Gaussian amplitude. A small non-Gaussian wing, with a minor contribution to the TDCS, was previously observed in DPI measurements on $\mathrm{Ne} 2 s$ [32] and attributed to intershell $(2 s, 2 p)$ mixing in the initial state. At variance in the present measurements an extended wing, which provides a dominant contribution to the TDCS, is observed.

Interference in the angular distributions of DPI has been considered previously. However, the interference effect discussed here is of a completely different nature. For example in indirect DPI, where the process proceeds via the formation of an intermediate singly charged ion state, when the photoelectron and the Auger electron have the same kinetic energy, and therefore are indistinguishable, interference was predicted [33] and observed [34] at certain mutual angles $\theta_{12}$. Interference between the various partial waves of the electron pair in the continuum has been also predicted in the analysis of the TDCS in terms of a bipolar harmonics expansion used to fit the Ca TDCS [35]. The interference referred to here is more similar to the one of the electron "two-slit" experiments. There the wave functions of the electrons, "emitted" from different spatial positions, interfere. Here, similarly, the wave functions of two-electron pairs "originated" from two different regions of the charge density interfere. This effect is well described by a representation of the gerade amplitude by a di-Gaussian function.

In summary the measurement of the DPI of a $\mathrm{Mg} 3 s$ orbital in the equal energy sharing condition has shown that the amplitude, which describes the correlated angular distribution of the electron pair, is strongly determined by the extent of the radial wave function of the target orbital. This is similar to the Cooper minimum in single photoionization cross sections. However, the weaker constraints on the angular momenta of the electron pair result in a complex interference pattern instead of a simple minimum. Just as the Cooper minimum is a universal phenomenon, the breaking down of the Gaussian ansatz in describing the symmetric DPI amplitude should be found in all quasitwo-electron targets beyond helium.

This work was partially supported by the MIUR PRIN 2009W2W4YF and 2009SLKFEX and the SFI Grant No. 08/RFP/PHY1117. The authors thank P. Bertoch and F. Salvador for prompt and qualified technical assistance in assembling the $\mathrm{Mg}$ oven at Elettra. Resources of the Australian NCI Facility were used in this work.

[1] U. Fano and J. W. Cooper, Rev. Mod. Phys. 40, 441 (1968).

[2] J. B. Bertrand, H. Wörner, P. Hockett, D. Villeneuve, and P. Corkum, Phys. Rev. Lett. 109, 143001 (2012).

[3] S. L. Molodtsov, S. Halilov, M. Richter, A. Zangwill, and C. Laubschat, Phys. Rev. Lett. 87, 017601 (2001).

[4] D. V. Vyalikh et al., Phys. Rev. Lett. 100, 056402 (2008).

[5] A.S. Kheifets, I. Bray, J. Colgan, and M. S. Pindzola, J. Phys. B 44, 011002 (2011).

[6] L. Avaldi and A. Huetz, J. Phys. B 38, S861 (2005).

[7] J. S. Briggs and V. Schmidt, J. Phys. B 33, R1 (2000).

[8] L. Malegat, Phys. Scr. T110, 83 (2004). 
[9] O. Schwarzkopf, B. Krässig, J. Elmiger, and V. Schmidt, Phys. Rev. Lett. 70, 3008 (1993).

[10] A.S. Kheifets and I. Bray, Phys. Rev. A 65, 012710 (2001).

[11] F. Citrini, L. Malegat, P. Selles, and A. K. Kazansky, Phys. Rev. A 67, 042709 (2003).

[12] J. Colgan and M. S. Pindzola, Phys. Rev. A 65, 022709 (2002).

[13] S. C. Ceraulo, R. M. Stehman, and R. S. Berry, Phys. Rev. A 49, 1730 (1994).

[14] A. Kazansky and V. N. Ostrovsky, J. Phys. B 30, L835 (1997).

[15] F. Maulbetsch, I. L. Cooper, and A. S. Dickinson, J. Phys. B 33, L119 (2000).

[16] L. Malegat, F. Citrini, P. Selles, and P. Archirel, J. Phys. B 33, 2409 (2000).

[17] A. S. Kheifets and I. Bray, Phys. Rev. A 75, 042703 (2007).

[18] P. Bolognesi, M. Coreno, G. Alberti, R. Richter, R. Sankari, and L. Avaldi, J. Electron Spectrosc. Relat. Phenom. 141, 105 (2004).

[19] R. R. Blyth et al., J. Electron Spectrosc. Relat. Phenom. 101-103, 959 (1999).

[20] See Supplemental Material at http://link.aps.org/ supplemental/10.1103/PhysRevLett.110.083001 for details of the experimental setup and of the theoretical model.

[21] K. J. Ross and J. B. West, Meas. Sci. Technol. 9, 1236 (1998).
[22] H.-J. Beyer, J.B. West, K. J. Ross, and A. De Fanis, J. Phys. B 33, L767 (2000).

[23] K. J. Ross, J. B. West, and H.-J. Beyer, J. Phys. B 30, L735 (1997).

[24] K. J. Ross, J.B. West, H.-J. Beyer, and A. De Fanis, J. Phys. B 32, 2927 (1999).

[25] J.B. West, K. J. Ross, H.-J. Beyer, A. De Fanis, and H. Hamdy, J. Phys. B 34, 4167 (2001).

[26] P. Sheridan, M. Grimm, and E. Sokell, J. Phys. B 41, 165204 (2008).

[27] M. Y. Amusia and A. S. Kheifets, Phys. Lett. A 82, 407 (1981).

[28] U. Fano, Phys. Rev. 124, 1866 (1961).

[29] R. Wehlitz and P. N. Juranić, Phys. Rev. A 79, 013410 (2009).

[30] M. Stener, G. De Alti, G. Fronzoni, and P. Decleva, Chem. Phys. 222, 197 (1997).

[31] P. Bolognesi et al., Phys. Scr. T110, 62 (2004).

[32] P. Bolognesi, R. Flammini, A. Kheifets, I. Bray, and L. Avaldi, Phys. Rev. A 70, 062715 (2004).

[33] L. Vegh and J. H. Macek, Phys. Rev. A 50, 4031 (1994).

[34] S. Rioual, B. Rouvellou, L. Avaldi, G. Battera, R. Camilloni, G. Stefani, and G. Turri, Phys. Rev. Lett. 86, 1470 (2001).

[35] F. Maulbetsch, I. L. Cooper, and A. S. Dickinson, J. Phys. B 33, L119 (2000). 